\title{
Differenzialtherapie im Alter am Beispiel der Arteriellen Hypertonie
}

Für die medikamentöse Behandlung Erwachsener spielt das Alter oft eine große Rolle. So werden zunehmend Studien mit älteren Teilnehmern gefordert, um die bei jüngeren Menschen gesehenen Effekte dort zu replizieren. Beispiele hierfür sind die Behandlung der arteriellen Hypertonie oder die Senkung der Blutfette in der Primär- oder Sekundärprävention.

Im klinischen Alltag spielt allerdings oft weniger das Alter, als vielmehr die körperliche Gebrechlichkeit („Frailty“) eine wichtige Rolle bei der Abwägung von möglichem Nutzen und Schaden einer Therapieoption. Je nach Wirkung- und Nebenwirkungsausmaß kann sich die Therapie-Entscheidung bereits bei leichter oder bei schwerer Frailty ändern.

Frailty wird im medizinischen Alltag oft durch das bloße Augenmaß bestimmt, obwohl unterschiedliche Instrumente existieren, die eine Quantifizierung und Qualifizierung ermöglichen. Auch in klinischen Studien fehlen fast immer geeignete Assessments, um einzuschätzen, ob auch gebrechliche Patienten in den Kohorten zu finden sind. Somit stellt eine kritische Bewertung des Behandlungserfolgs in dieser Population eine große Herausforderung dar.

Um hier weiter zu kommen, wollten wir einen Weg finden, Patientenkohorten in Studien mit Einschluss älterer Teilnehmer (65 Jahre und älter) retrospektiv besser einzuordnen, um zumindest einen indirekten Vergleich der Therapieevidenz zu ermöglichen. Dazu haben wir im Rahmen mehrerer systematischer Übersichtsarbeiten von randomisierten und nicht-randomisierten kontrollierten klinischen Studien sowie Beobachtungsstudien bei älteren Menschen mit arterieller Hypertonie, Diabetes mellitus, Demenz und Depression zunächst alle in diesen Studien angewendete und für eine Beurteilung des Funktionszustands der Teilnehmer geeignete Assessments identifiziert.

Ergänzt wurde die Liste der gefundenen Assessments durch eine nicht-systematische Literatursuche nach häufig verwendeten Gebrechlichkeits- und funktionellen
Skalen. Es erfolgte eine Unterteilung des körperlichen Funktionszustands in vier Stufen.

Daraufhin wurden die Assessments entsprechend analysiert und nach einer einfachen, Patienten-zentrierten Berechnungsmethode zu den vier Stufen gehörige Grenzwerte ermittelt. Diese folgt im Wesentlichen der Überlegung und Entscheidung, welches bestmögliche Ergebnis/ welche bestmögliche Antwort eine untersuchte/befragte Person einer bestimmten Funktionalitätskategorie optimalerweise erzielen könnte. Teilweise musste auch auf in der Literatur verfügbare Grenzwerte zurückgegriffen werden, wenn eine Berechnung nach der beschriebenen Methode nicht möglich war.

Es konnten 51 Assessment-Instrumente identifiziert werden, die in ausreichendem Maße (zu mindestens 25 Prozent) Messungen des körperlichen Funktionsstatus beinhalten. Klassische Gebrechlichkeits(Frailty)-Assessments wurden eingeschlossen, wenn sie ihren Schwerpunkt auf die Ermittlung körperlicher Gebrechlichkeit legten. Die den jeweiligen Kategorien zugewiesenen Grenzwerte sollen lediglich als Anhaltspunkte dienen, um Evidenz vorhandener Studienergebnisse bezüglich der Therapie von Menschen mit Funktionseinschränkungen besser identifizieren und beurteilen zu können [1].

Auf Basis dieser Annahmen erfolgten nun mehrere Reviews zu verschiedenen typischen Alterserkrankungen, von denen das Thema der medikamentösen Behandlung der arteriellen Hypertonie bereits publiziert werden konnte und hier kurz vorgestellt wird. Eine detaillierte Beschreibung des Forschungsvorhabens findet sich im PROSPERO-Protokoll Nummer CRD42017067253.

Für die Suchstrategie wurden validierte Filter für geriatrische Patienten angewendet. Diese wurden durch Begriffe zur Funktionalität und Hypertonie ergänzt. Zusätzlich wurden die Referenzlisten nationaler und internationaler Leitlinien sowie eingeschlossener Studien inklusive weiterer Zitate der Erst- und Letztautoren durch- sucht. Mögliche Vergleiche waren Antihypertensiva versus Placebo, keine oder eine andere medikamentöse Behandlung. Vergleiche verschiedener Blutdruckziele wurden ebenso eingeschlossen. Beobachtungsstudien, die ausschließlich die gemessenen Blutdruckwerte als Einflussvariable untersuchten, wurden ausgeschlossen.

Durch die große Anzahl an verwendeten Suchbegriffen zu Gebrechlichkeit, Alter und Hypertonie war der Screeningaufwand sehr hoch. Deshalb wurde die Literatur mit Hilfe von Covidence analysiert und selektiert, einer Screening-Software der Cochrane Collaboration, die durch verschiedene Funktionen den Auswahlprozess erleichtert und beschleunigt.

Dabei konnten insgesamt vier RCTs und drei Beobachtungsstudien in die Auswertung eingeschlossen werden. Die eingeschlossenen RCTs zeigen tendenziell, dass auch körperlich gebrechliche Patienten von der pharmakologischen Behandlung der Hypertonie im Hinblick auf Mortalität, kardiovaskuläre Erkrankungen, Funktionalität und Lebensqualität profitieren könnten. Demgegenüber weisen die Beobachtungsstudien zum Teil auf eine geringere Sturzrate und Sterblichkeit ohne antihypertensive Behandlung hin. Mit Blick auf die zwei wichtigsten Studien, SPRINT und HYVET, erscheint selbst bei funktionell eingeschränkten Patienten eine leitliniengerechte Blutdrucktherapie aber sinnvoll. Leider sind beide Studien aufgrund von Ausschlusskriterien nicht repräsentativ. Im Bereich der Non-RCTs findet man ebenfalls nur wenige Studien, die sowohl die medikamentöse Behandlung als auch körperliche Funktion differenziert analysieren. Somit empfehlen wir, bei älteren Menschen über 80 Jahre einen Blutdruck-Zielwert von < 140/90 mm Hg anzustreben, bei gebrechlichen Menschen aufgrund der HYVET Studie aber einen systolischen Zielwert von < 150 mm Hg [2].

Hier kann als einfaches Frailty Assessment etwa die Gehgeschwindigkeit herangezogen werden (Cut-off $0,8 \mathrm{~m} / \mathrm{s}$ ) - es sei denn, es handelt sich um Menschen mit sehr schlechter Funktionalität bzw. aus- 
geprägter körperlicher Gebrechlichkeit und damit voraussichtlich kurzer Lebenserwartung von weniger als 2 Jahren. Diese Patienten waren sicher nicht in den Studien eingeschlossen. Diese Vorschläge wurden von uns in einem Übersichtsartikel zur arteriellen Hypertonie im Alter im Rahmen einer CME-zertifizierten Fortbildung unter dem Titel „Arterielle Hypertonie im Alter mit dem Fokus 80+“(32) publiziert [3].

Schlussfolgerung: Aufgrund der unterschiedlichen Ergebnisse zwischen RCTs und non-RCTs, der geringen Repräsentation gebrechlicher Teilnehmer in den RCTs und eines hohen Verzerrungsrisikos in den non-RCTs sind die Resultate der eingeschlossenen Studien für die Ableitung von streng evidenzbasierten Therapieempfehlungen nicht geeignet. Als Richtwert schlagen wir vor, bei einer Gehgeschwindigkeit $<0,8 \mathrm{~m} / \mathrm{s}$ einen systolischen Blutdruck von < $150 \mathrm{~mm} \mathrm{Hg}$ anzustreben; be nicht oder nur leicht gebrechlichen Patienten $>80$ Jahre einen Wert von 130 $139 \mathrm{~mm} \mathrm{Hg}$.
Danksagung

Anmerkung: Mein besonderer Dank gilt Simone Brefka, Dhayana Dallmeier und Viktoria Mühlbauer sowie weiteren beteiligten Kolleginnen und Kollegen bei der Mitarbeit im Projekt Medikation und Lebenssituation im Alter.

\section{Interessenkonflikt}

Ich habe keine Interessenkonflikte im Zusammenhang mit den hier dargestellten Themen.

\section{Autorinnen/Autoren}

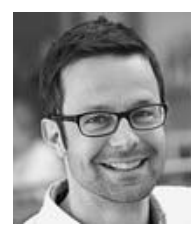

\section{Michael Denkinger}

apl. Prof. Dr. med., Ärztlicher Direktor der Agaplesion Bethesda Klinik und Vorsitzender Geriatrisches Zentrum Ulm/Alb-Donau und Universität Ulm
Korrespondenzadresse

\section{Prof. Michael Denkinger}

Geriatrische Forschung der Universität Ulm an der Agaplesion Bethesda Klinik Ulm Zollernring 26

$89073 \mathrm{Ulm}$

michael.denkinger@bethesda-ulm.de

\section{Literatur}

[1] Brefka S, Dallmeier D, Mühlbauer $V$ et al.; Medication and Quality of Life Research Group. A Proposal for the Retrospective Identification and Categorization of Older People With Functional Impairments in Scientific Studies-Recommendations of the Medication and Quality of Life in Frail Older Persons (MedQoL) Research Group. J Am Med Dir Assoc. 2019; 20: 138-146. doi:10.1016/j.jamda.2018.11.008

[2] Mühlbauer V, Dallmeier D, Brefka S et al. The Pharmacological Treatment of Arterial Hypertension in Frail, Older Patients - a Systematic Review. Dtsch Arztebl Int 2019; 116: 23-30. doi:10.3238/arztebl.2019.0023

[3] Dallmeier D, Denkinger MD. [Arterial hypertension in old age with the focus on $80+]$. Z Gerontol Geriatr. 2018; 51: 825-838. doi:10.1007/s00391-018-1454-0 